\title{
Following A Dynamic Object Through A Transient Response Adjustable MPC (Extended Version)
}

This paper was downloaded from TechRxiv (https://www.techrxiv.org).

LICENSE

CC BY-NC-SA 4.0

SUBMISSION DATE / POSTED DATE

$30-07-2020$ / 26-07-2021

\section{CITATION}

Hong, Sanghyun; Miller, Justin; Lu, Jianbo (2020): Following A Dynamic Object Through A Transient Response Adjustable MPC (Extended Version). TechRxiv. Preprint.

https://doi.org/10.36227/techrxiv.12740372.v2

$\mathrm{DOI}$

10.36227/techrxiv.12740372.v2 


\title{
Following A Dynamic Object Through A Transient Response Adjustable MPC (Extended Version)
}

\author{
Sanghyun Hong ${ }^{* \dagger}$, Justin Miller*, and Jianbo Lu*
}

\begin{abstract}
Robots can help humans fulfill various tasks in efficient and safe manners. For example, robots can navigate while loaded with packages to assist humans in delivery tasks, and they can also collaborate with Autonomous Vehicles (AV) to reach areas that AVs cannot drive. As robots are expected to interact with humans, vehicles, and other robots, following these dynamic objects is considered a crucial functionality. This paper will propose a mobile robot motion controller for following a dynamic object based on downscaled Model Predictive Control (MPC). It can reflect the dynamics of object for an appropriate response speed and ensure safety of mobile robot against predicted risks. In addition, an adaptive prediction time horizon will be proposed to improve robustness in following a dynamic object. The proposed motion controller is demonstrated through extensive simulations and real-time experiments of a test mobile robot.
\end{abstract}

\section{INTRODUCTION}

Human-robot interaction has gained increasing attention, allowing humans to fulfill tasks quickly and safely through assistance of robots. In order for mobile robots to interact with humans, a crucial functionality is following a person in a way that ensures efficient human-robot interaction. For example, if a mobile robot follows a delivery person with packages on it, following distance should be within a reasonable range for delivery efficiency and the mobile robot should be able to regulate its maneuvers to prevent collision with obstacles and rollover caused by a high mass center. In addition to humanrobot interaction, following functionality is also important when a mobile robot needs to collaborate with another mobile robot, e.g., mobile robot platooning for mass package delivery.

To follow an object, mobile robots need an object tracking algorithm that recognizes the location of the object and a motion control algorithm that creates maneuvers that will follow the object in an appropriate manner. There have been various motion control algorithms proposed for following a person. Lee et al. [1] used Dynamic Window Approach (DWA) within the Robot Operating System (ROS) navigation stack. A drawback to that approach is that the prediction of the trajectory is limited because of a short prediction time horizon. Moreover, safe maneuvering of the mobile robot for rollover prevention and correct following of the planned path were not taken into consideration in its baseline algorithm. They had to implement additional control schemes to compensate for limitations of DWA. Doisy et al. [2] utilized a path following algorithm that applied a discontinuous feedback control law

$\dagger$ Corresponding author

* S. Hong, J. Miller, and J. Lu are with Research \& Advanced Engineering, Ford Motor Company, Dearborn, MI, USA (e-mail: \{shong7; jmill597; jlu10\}@ford.com). proposed by Morin et al. [3], which is known as Sliding Mode Control (SMC). Shanee et al. [4] implemented Proportional Control (P-Control) along with the SMC for path following algorithm in order to follow a person with a pre-defined angle. However, their approach considered neither the response speed to the motion of persons nor safe maneuvering of mobile robots. Chen et al. [5] developed a vision-based person following in which a simple proportional control was used without accounting for preventing rollover of mobile robots and collisions with obstacles. Olmedo et al. [6] developed a people following mobile robot with laser scanners, but it is based on PID control which does not take safety of the mobile robot and optimality of control into consideration. Although Leigh et al. [7] extended the methodology utilizing laser scanners so that mobile robots can track legs of person, they employed the same PID controller for following persons.

In following a highly dynamic target, e.g., a human, it is important to find optimal control commands that balance the trade-off between navigation cost (e.g., time and actuation) and safety (e.g., collision avoidance, prevention of packages fall off, and rollover prevention with packages loaded) before any potential risk is imminent. Therefore, among the various motion control schemes for following an object, our scope will be Model Predictive Control (MPC) framework. There are several motion control approaches based on the predictive optimal control framework for mobile robots, e.g., Dynamic Window Approach (DWA) [8] and Trajectory Rollout (TR) [9]. However, they mainly focus on reaching a goal without taking into account package fall off and rollover prevention, transient response speed, or tight path following.

This paper develops an MPC-based motion controller for following a dynamic object that accounts for rollover prevention, collision avoidance, and response speed. We will perform theoretical analysis of the proposed MPC formulation to prove that the prediction time horizon can be used to determine transient response speed. In other words, the proposed motion controller can adjust prediction time horizon to reflect dynamics of the object followed by mobile robots. In addition, we will propose an adaptive prediction time horizon to ensure robustness in following a dynamic object. The proposed motion control approach will be demonstrated through extensive simulation and experimental tests.

\section{Problem Definition}

The problem to be addressed in this paper is related to how a mobile robot is following a dynamic object. The solution to this problem has application in various domains, e.g., package 
delivery and personal assistance. Consider the case shown in Fig. 1, where a blue mobile robot is tasked to follow the orange mobile robot by tracking its relative location. Once the blue robot detects the orange robot, it sets a goal with an offset $d_{\text {off }}$ from the orange robot, as indicated by the yellow circle in Fig. 1. Then, the blue mobile robot follows the goals that are published whenever a time period or minimum displacement is met as the orange robot moves.

The blue robot computes control commands, $u$, in the form of linear and angular velocities, e.g., longitudinal velocity and yaw rate, that will depend on the distance, $d(k)$, from the blue robot to the goal at time $k$. In Fig. 1, the length of arrows represents the magnitude of the computed linear velocity. Additionally, there are constraints on the linear and angular accelerations, i.e. the upper bound on the magnitude of acceleration, to prevent excessively aggressive motion that can cause a dangerous situation, e.g., collision with the orange robot. In order to follow a highly dynamic object, e.g., a
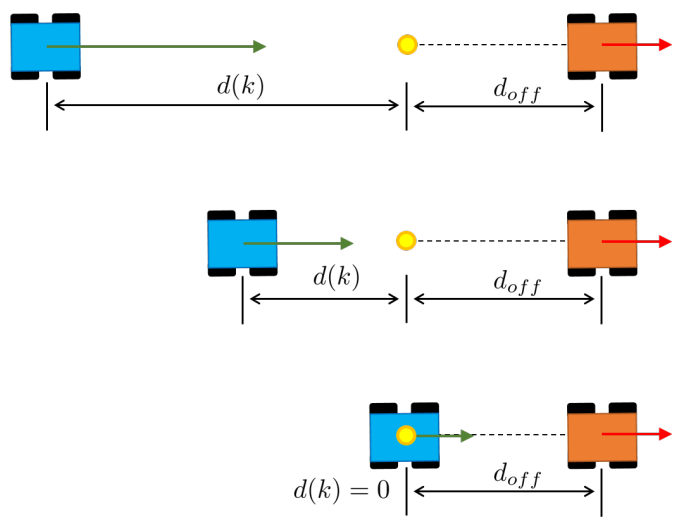

Figure 1: A Mobile Robot Following A Dynamic Object

human, that changes maneuvers continuously and drastically, the wheeled mobile robot should be able to account for the response speed for the highly dynamic motion of the object. In addition, the mobile robot should have the capability to avoid collisions with obstacles and prevent loss of control like rollover caused by high mass center with packages loaded.

\section{MPC FORMULATION FOR FOLLOWING MOTION CONTROL}

When following a dynamic object, if the object changes maneuvers, a mobile robot needs to respond to the changes of motion by accelerating linear and angular velocities so that it does not lose track of the dynamic object. In addition, if a dynamic object stays at a steady state, a mobile robot also needs to find appropriate navigation velocities to remain within an appropriate distance from the object. Meanwhile, if any potential risks, e.g., collision and rollover, are anticipated, the mobile robot should prioritize safety and compute control commands to prevent dangerous situations at the cost of causing the mobile robot to fall behind. This implies that there exists trade-offs between following distance and safety, thus leading to the need for an optimal control method. In addition, taking action preemptively against potential risks is the best way to prevent collision with obstacles, package fall-off, and robot rollover, which requires looking ahead. This leads us to prediction of motion in the motion control scheme.

Therefore, we will use Model Predictive Control (MPC), also known as receding horizon optimal control, to develop a motion control algorithm that follows a dynamic object with safety ensured. With the MPC framework, a constrained optimal control problem is solved at time step $k$ to obtain a sequence of optimal control commands, i.e. $u(k), \ldots, u(k+N)$, where $N$ represents the prediction time horizon. Once the optimal control commands are computed, only the first command $u(k)$ will be applied to mobile robot to generate a desired maneuver. Then, the above procedures are repeated at every time step afterwards.

In this paper, we share the MPC framework with the exiting predictive motion control approaches, e.g., DWA and TR, but our formulation will consider safe motion to prevent rollover and package fall-off, tight path following, collision avoidance, and realistic accelerations. The constrained optimal control problem for the proposed MPC-based motion controller is defined as follows.

$$
\begin{array}{cl}
\min _{U} & h\left(\{s(j)\}_{j=k_{c}}^{k_{c}+N}, U\right) \\
\text { subj. to } & s(k+1)=f(s(k), u(k)) \\
& \ddot{\psi}^{l b} \leq \ddot{\psi}\left(k_{c}+j\right) \leq \ddot{\psi}^{u b} \\
& a_{x}^{l b} \leq a_{x}\left(k_{c}+j\right) \leq a_{x}^{u b} \\
& a_{y}^{l b} \leq a_{y}\left(k_{c}+j\right) \leq a_{y}^{u b} \\
& \text { for } j=0,1, \cdots, N,
\end{array}
$$

where $k_{c}$ represents the time step at which the above problem is solved and $N$ represents the time horizon for prediction. In (1), the constrained optimal control problem minimizes a cost function $h(\cdot)$ in (1a) which is a function of a predicted sequence of system states $\{s(j)\}_{j=k_{c}}^{k_{c}+N}$ and control inputs $U=\{u(j)\}_{j=k_{c}}^{k_{c}+N}=\left[u\left(k_{c}\right), \ldots, u\left(k_{c}+N\right)\right]$. The system, i.e., a mobile robot, state $s(k)$ evolves based on the system dynamic equation $f(\cdot)$ in (1b). Accelerations in linear and rotational motion, i.e., $a_{x}(k), a_{y}(k)$ and $\ddot{\psi}(k)$, are constrained by lower and upper bounds in (1c)-(1e), where the superscripts $l b$ and $u b$ represent lower bound and upper bound, respectively. As shown in Fig. 2, the subscripts $x$ and $y$ represent the longitudinal and lateral directions in the local frame of mobile robot, and $\psi$ represents the rotational motion about $z$ axis pointing toward readers, i.e. yaw motion. The global frame

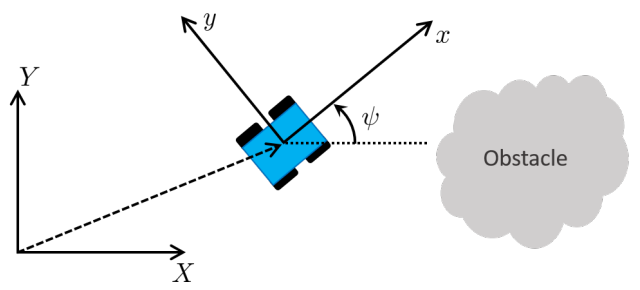

Figure 2: A Global Frame and A Local Frame of Mobile Robot

$O X Y Z$ in Fig. 2 is an inertial frame that is fixed with a constant orientation. 


\section{A. Mobile Robot System Dynamics}

In this subsection, we define the state $s$ for the mobile robot in (1b) that consists of the coordinate $X$ and $Y$ in the global frame, yaw/heading angle $\psi$, longitudinal velocity $v_{x}$, lateral velocity $v_{y}$, and yaw rate $\dot{\psi}$, i.e., $s=\left[X, Y, \psi, v_{x}, v_{y}, \dot{\psi}\right]^{\top}$. With the sampling time $\tau$, the dynamic state space representation $f(\cdot)$ in $(1 \mathrm{~b})$ is constructed as follows.

$$
\left[\begin{array}{c}
X(k+1) \\
Y(k+1) \\
\psi(k+1) \\
v_{x}(k+1) \\
v_{y}(k+1) \\
\dot{\psi}(k+1)
\end{array}\right]=\left[\begin{array}{l}
X(k)+\tau v_{X}(k) \\
Y(k)+\tau v_{Y}(k) \\
\psi(k)+\tau \dot{\psi}(k) \\
v_{x}(k)+\tau \dot{v}_{x}(k) \\
v_{y}(k)+\tau \dot{v}_{y}(k) \\
\dot{\psi}(k)+\tau \ddot{\psi}(k)
\end{array}\right],
$$

where

$$
\begin{aligned}
& {\left[\begin{array}{l}
v_{X}(k) \\
v_{Y}(k) \\
v_{x}(k) \\
v_{y}(k)
\end{array}\right]} \\
& \quad=\left[\begin{array}{l}
v_{x}(k) \cos \psi(k)-v_{y}(k) \sin \psi(k) \\
v_{x}(k) \sin \psi(k)+v_{y}(k) \cos \psi(k) \\
a_{x}(k)+v_{y}(k) \dot{\psi}(k) \\
a_{y}(k)-v_{x}(k) \dot{\psi}(k)
\end{array}\right] .
\end{aligned}
$$

In the above equations, $v_{X}$ and $v_{Y}$ represent the velocities in the $X$ and $Y$ direction of global frame, respectively. In addition, $\dot{v}_{x}$ and $\dot{v}_{y}$ represent the time derivatives of the longitudinal velocity $v_{x}$ and lateral velocity $v_{y}$ of the mobile robot, respectively. Readers are referred to [10] for derivation of the above dynamic equations.

Note that this paper will use a nonholonomic mobile robot in Simulation Results and Experimental Results, and therefore $v_{y}$ and $v_{y}$ in (2) will be set to zeros.

\section{B. Cost Function}

The cost function to be minimized is defined with the predicted states and control commands as follows.

$$
\begin{aligned}
& h\left(\{s(j)\}_{j=k_{c}}^{k_{c}+N}, U\right) \\
& =\omega_{o} \max \left(\left\{h_{o}\left(d_{o}(i)\right)^{2}\right\}_{i=k_{c}}^{k_{c}+N}\right) \\
& \quad+\omega_{a y} \max \left(\left\{a_{y}(i)^{2} / \bar{a}_{y}(i)^{2}\right\}_{i=k_{c}}^{k_{c}+N}\right) \\
& \quad+\omega_{g} d_{g}\left(k_{c}+N\right)^{2} \\
& \quad+\omega_{p} \sum_{j=0}^{N} d_{p}\left(k_{c}+j\right)^{2},
\end{aligned}
$$

where $\omega_{o}, \omega_{a y}, \omega_{g}$, and $\omega_{p}$ are weights.

The first cost term in (3a) enables avoiding collisions with obstacles. The function $h_{o}$ is a function that is inversely proportional to $d_{o}(k)$ which is the distance at time $k$ from the mobile robot to an obstacle, as shown in Fig. 3a. Therefore, the term in (3a) accounts for the location where the mobile robot navigates closest to an obstacle along the predicted trajectory. The predicted trajectory is obtained from the sequence of the first and second state variables, $X$ and $Y$. In Fig. 3a, the magenta line represents the path planned by a path planner from the mobile robot to a goal, and the green line is the predicted trajectory.

In order to ensure the safety of the mobile robot, i.e., prevent rollover and package fall-off, we consider the largest ratio of the lateral acceleration $a_{y}(\cdot)$ to the allowed maximum lateral acceleration $\bar{a}_{y}(\cdot)$, which are calculated along the predicted trajectory. The $\bar{a}_{y}(\cdot)$ is a pre-defined lateral acceleration profile as a function of $v_{x}$, e.g., piecewise linear function of $v_{x}$ in which $\bar{a}_{y}=2 \mathrm{~m} / \mathrm{s}^{2}$ for $v_{x}<0.5 \mathrm{~m} / \mathrm{s}$ and $\bar{a}_{y}=1 \mathrm{~m} / \mathrm{s}^{2}$ for $v_{x} \geq 0.5 \mathrm{~m} / \mathrm{s}$. If the mobile robot does not slow down in a sharp turn while following an object, it may lose contact with the ground and slide. Furthermore, if the mobile robot follows an object with packages loaded, it may cause the packages to fall when taking a sharp turn that generates a large lateral acceleration. Therefore, the lateral acceleration is included in the cost function for safe maneuvering of the mobile robot. With a nonholonomic mobile robot, we can obtain $a_{y}$ from the fourth element in (2b) by substituting zero to $\dot{v}_{y}$ as $a_{y}=v_{x} \dot{\psi}$.

To head for a goal, the distance to the goal $d_{g}$ is included in the cost, as presented in (3c). Note that the distance to the goal at the end of the predicted trajectory is used, as shown in Fig. 3b, and the distance is not a straight airline distance to account for the distance required to navigate without collision with obstacles.

The fourth term in (3d) contains the distance from the mobile robot to the planned path $d_{p}$, as shown in Fig. 3c, and therefore, this cost term is utilized for tight path following. The path is planned by a path planning algorithm, e.g., $A^{*}$ algorithm [11], and the details are not discussed herein since it is beyond the scope of this paper. A planned path is commonly used as guidance for a mobile robot to navigate. Therefore, if a path is planned periodically to reflect a dynamic environment, following the planned path helps the mobile robot to navigate by avoiding collisions with obstacles. This is the reason that we keep a path planner on top of the motion controller predicting trajectories.

\section{Accelerations to Track Constant Reference Velocities}

In the previous subsection, we have defined different cost function from the existing predictive mobile robot motion control algorithms, e.g., Dynamic Window Approach (DWA) [8] and Trajectory Rollout (TR) [9]. But, the decision variables in (1) will be defined similarly to downscale the constrained optimal control problem in (1).

In general, the decision variables in (1) would be the sequence of accelerations in linear and rotational motion, $U=\left[\left\{a_{x}(j)\right\}_{j=k_{c}}^{k_{c}+N},\left\{a_{y}(j)\right\}_{j=k_{c}}^{k_{c}+N},\{\ddot{\psi}(j)\}_{j=k_{c}}^{k_{c}+N}\right]$, which is a vector in the dimension of $3(N+1)$. But, in this paper, we utilize the steady state reference tracking control [12] to define the accelerations such that the mobile robot can converge to the constant reference velocities, $v_{x}^{\text {ref }}, v_{y}^{r e f}$, and $\dot{\psi}^{r e f}$, over the prediction time horizon $\tau_{h}=N \tau$, as follows.

$$
\left[\begin{array}{c}
a_{x}(k) \\
a_{y}(k) \\
\ddot{\psi}(k)
\end{array}\right]=\left[\begin{array}{c}
K_{x}\left(v_{x}^{r e f}-v_{x}(k)\right) \\
K_{y}\left(v_{y}^{r e f}-v_{y}(k)\right) \\
K_{\psi}\left(\dot{\psi}^{r e f}-\dot{\psi}(k)\right)
\end{array}\right]
$$




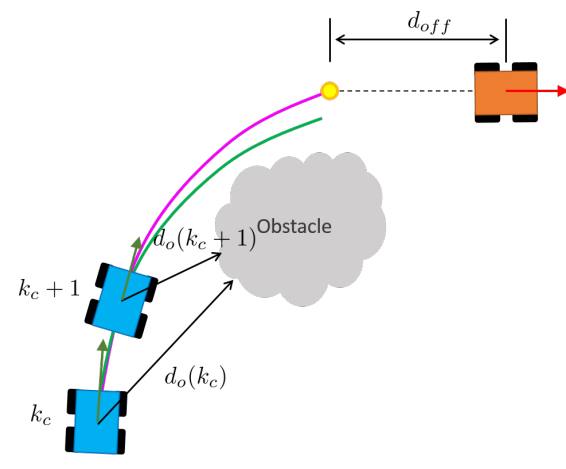

(a) Distance to obstacle

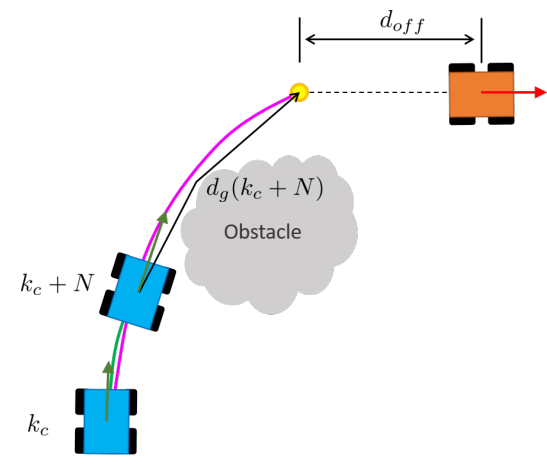

(b) Distance to goal

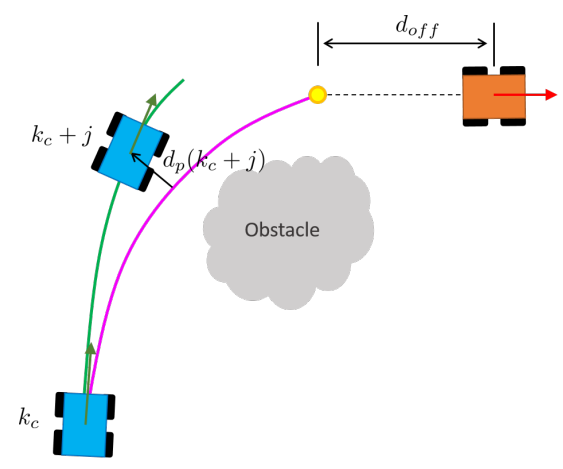

(c) Distance to path

Figure 3: Cost Function

where the feedback gains are

$$
K_{i}=\frac{1-p_{i}}{\tau}, \text { for } i \in\{x, y, \psi\} .
$$

In other words, with the above approach, we constrain the accelerations to functions of the constant reference velocities. In the definition of gain in (5), $p_{i}$ represents the closed loop pole that determines the rate of convergence, and its absolute value should be $\left|p_{i}\right|<1$ for stability. As the value of $p_{i}$ becomes smaller, the velocity of the mobile robot converges to the reference one faster.

By substituting (4) and (5) into the control inputs $U$ in (1), the decision vector is downscaled to $U=\left[v_{x}^{\text {ref }}, v_{y}^{\text {ref }}, \dot{\psi}^{\text {ref }}\right]$, which is in the 3-dimensional space, since $v_{x}(k), v_{y}(k)$, and $\dot{\psi}(k)$ in (4) are obtained as the state variables. Therefore, the problem in (1) can be solved with a significantly reduced computational cost.

If $p_{i}$ is zero in (5), the mobile robot achieves the constant reference velocity immediately after one sampling time $\tau$, and the reference velocity is maintained for the entire prediction time horizon $\tau_{h}$ since the accelerations become zero after one sampling time. Although the immediate convergence to the reference velocities with $p_{i}=0$ is not realistic, it has been assumed in DWA [8] and TR [9] algorithms. However, this paper will use nonzero $p_{i}$ in simulations and experiments.

\section{Solving the Downscaled MPC}

We solve the downscaled MPC in (1) by searching a discretized control space. Therefore, $n_{i}$ values are sampled from each decision variable, and a search space is constructed with all possible combinations of the sampled values. Then, by searching for the grid point in the search space that generates the smallest cost in (3), the optimal combination of decision variable values can be found.

In this paper, due to (4), the decision variables are $U=$ $\left[v_{x}^{r e f}, v_{y}^{r e f}, \dot{\psi}^{r e f}\right]$. Therefore, for example, we sample $n_{v x}$ values spacing the range of $\left[v_{x}(k)-\tau_{h} a_{x}^{l b}, v_{x}(k)+\tau_{h} a_{x}^{u b}\right]$ for the decision variable $v_{x}^{r e f}$. In other words, the $i^{\text {th }}$ sample for $v_{x}^{r e f}$ is

$$
v_{x}-\tau_{h} a_{x}^{l b}+\frac{\tau_{h}\left(a_{x}^{l b}+a_{x}^{u b}\right)}{n_{v x}} i .
$$

Likewise, $n_{v y}$ and $n_{\psi}$ values are sampled for the decision variables $v_{y}^{\text {ref }}$ and $\psi^{\text {ref }}$, respectively. If a nonholonomic mobile robot is used, we sample values from $v_{x}^{r e f}$ and $\dot{\psi}^{\text {ref }}$ only, and the optimal decision variables $v_{x}^{*}$ and $\dot{\psi}^{*}$ are found by evaluating costs for the sampled values.

With the above approach, the MPC in (1) can be solved without an optimization solver. Most optimization solvers assume the cost function is differentiable, and therefore if we do not use an optimization solver, a non-differentiable cost function can be formulated. In addition, we can guarantee a global minima within the search space. If we define an MPC with many decision variables and many sample values are required for the decision variables, this approach is not feasible in real time and an optimization solver may be necessary. But, we have the 3 dimensional decision vector, and, in practice, with mobile robots, we do not have to search for $v_{x}^{\text {ref }}$ values at very fine resolutions, e.g., $0.01 \mathrm{~m} / \mathrm{s}$. Therefore, we can solve the MPC in (1) in real time with a reasonably small number of samples for the decision variables.

\section{Transient Response Analysis and Adaptive PREDICTION TIME HORIZON}

\section{A. Transient Response of Mobile Robots}

With the accelerations constrained to converge to constant reference velocities in the downscaled MPC in (1), it is found out that the prediction time horizon can be used to adjust transient response in following a dynamic object, as presented below. Therefore, the prediction time horizon provides a way to determine how quickly a mobile robot responds to motion of a dynamic object.

Theorem IV.1. Suppose an MPC defined in the form of (1) whose cost function includes the distance to goal at the end of predicted trajectory, $d_{g}\left(k_{c}+N\right)$, where $k_{c}$ represents the time step at which the MPC problem is solved. If the linear accelerations, $a_{x}$ and $a_{y}$, are constrained to track constant reference velocities, $v_{x}^{r e f}$ and $v_{y}^{r e f}$, the time horizon for prediction, $\tau_{h}=\tau N$, acts as the time constant to determine transient response (see [13] for definition of time constant).

Proof. The above statement can be proved by direct proof. Without loss of generality, consider a mobile robot heading for 
a static goal on a straight line in the $\mathrm{X}$ direction of the global frame in Fig. 2, and it can generate the acceleration $a_{x}(k)$ to converge to the constant reference velocity $v_{x}^{r e f}$ immediately. In addition, cost terms other than the distance to a goal and acceleration constraints are excluded. Then, the downscaled MPC is formulated as follows.

$$
\begin{array}{ll}
\min _{v_{x}^{r e f}} & d_{g}\left(k_{c}+N\right) \\
\text { subj. to } & {\left[\begin{array}{l}
X(k+1) \\
v_{x}(k+1)
\end{array}\right]=\left[\begin{array}{l}
X(k)+\tau v_{x}(k) \\
v_{x}(k)+\tau a_{x}(k)
\end{array}\right],} \\
& \text { where } a_{x}(k)=\frac{1}{\tau}\left(v_{x}^{r e f}-v_{x}(k)\right) .
\end{array}
$$

If the above problem is solved at every time step with sampling time $\tau$, we always obtain the optimal constant reference velocity $v_{x}^{r e f}$ such that $v_{x}^{r e f} \tau_{h}=d_{g}\left(k_{c}\right)$, where $\tau_{h}=\tau N$ and $d_{g}\left(k_{c}\right)$ is the distance between robot and goal at the time when the above problem is solved. In other words, the mobile robot chooses $v_{x}^{r e f}$ such that it can travel the distance $d_{g}\left(k_{c}\right)$ with the constant velocity $v_{x}^{r e f}$ for the prediction time horizon $\tau_{h}$. This is because the above MPC minimizes the distance to goal from the end of the predicted trajectory, i.e. $d_{g}\left(k_{c}+N\right)$.

In the continuous time $t$ domain, by omitting the subscript $c$ for simplicity, we can rewrite as follows.

$$
v_{x}^{r e f} \tau_{h}=d_{g}(t)
$$

and since $v_{x}^{r e f}=\dot{X}(t)$,

$$
\dot{X}(t)=\frac{d_{g}(t)}{\tau_{h}}=\frac{X_{g}-X(t)}{\tau_{h}},
$$

where $X(t)$ and $X_{g}$ represent the coordinates of mobile robot at $t$ and goal in the $X$ axis of the global frame, respectively.

Applying the Laplace Transform to (7) with the assumption of zero initial condition yields,

$$
\begin{aligned}
s \mathcal{X}(s) & =\frac{1}{\tau_{h}} \frac{X_{g}}{s}-\frac{1}{\tau_{h}} \mathcal{X}(s) \\
\Rightarrow \quad\left(s+\frac{1}{\tau_{h}}\right) \mathcal{X}(s) & =\frac{1}{\tau_{h}} \frac{X_{g}}{s} \\
\Rightarrow \quad \mathcal{X}(s) & =\frac{X_{g} / \tau_{h}}{s\left(s+1 / \tau_{h}\right)},
\end{aligned}
$$

where the Laplace Transform of $X(t)$ is represented by $\mathcal{X}(s)=\mathcal{L}\{X(t)\}$ and $s$ represents the complex variable.

Then, the Inverse Laplace Transform of (8) yields

$$
X(t)=X_{g}\left(1-e^{-t / \tau_{h}}\right) .
$$

By the definition of time constant, the time at which $X(t)$ reaches around $(1-1 / e) \times 100 \approx 63.2 \%$ of $X_{g}$ is the prediction time horizon $\tau_{h}$, i.e., $X\left(\tau_{h}\right) \approx 0.632 X_{g}$. Therefore, the prediction time horizon $\tau_{h}$ is the time constant to determine transient response when navigating to a goal with the above MPC.

\section{B. Adaptive Prediction Time Horizon}

As presented in the previous subsection, the prediction time horizon $\tau_{h}$ can be tuned to determine transient response speed. This implies that the mobile robot can follow a dynamic object quickly and fast as the prediction time horizon decreases.
But, with the small $\tau_{h}$, the mobile robot can exhibit undesired behavior, e.g., occasional wiggle and stop, which causes a lost dynamic object. The small prediction time horizon generates short trajectories that make it difficult to find the optimal trajectory in terms of the associated cost value. In Fig. 4 , the distance to goal from the end of predicted trajectory is illustrated as the cost, and the black vertical line represents a wall. With a large $\tau_{h}$, the motion controller can predict trajectories long enough to determine the yellow one is the better choice, as shown in Fig. 4b. But, with a small $\tau_{h}$, we end up with two short trajectories that have almost same cost values, i.e., distance to goal at the end of trajectory, as shown in Fig. 4a. This can make the motion controller vulnerable to noise, which hinders from obtaining an optimal command and eventually degrades performance of following a dynamic object.

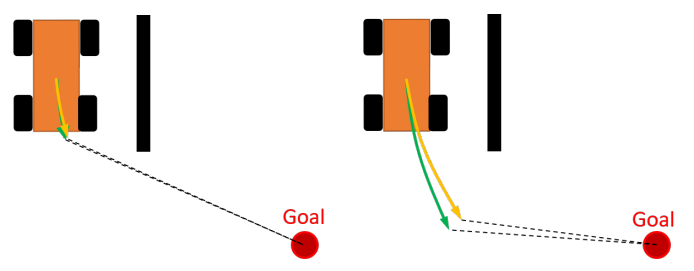

(a) Short trajectory prediction (b) Long trajectory prediction

Figure 4: Small prediction time horizon degrading following performance

Therefore, this paper proposes to make the prediction time horizon $\tau_{h}$ adaptive to the distance to goal. First, $\tau_{h}$ is set to be a small value, e.g., 0.5 second, to follow a dynamic object by reacting quickly to its movement. But, instead of using $\tau_{h}$ directly for motion control, we define a temporary time horizon $\tau_{h_{-} \text {temp }}$ as follows.

$$
\tau_{h_{-} t e m p}=\omega_{\tau} \frac{d_{g}(k)}{v_{x}(k)+\tau_{h}^{u b} a_{x}^{u b}},
$$

where $\omega_{\tau}$ is a scaling factor whose value is between 0.5 and $1, d_{g}(k)$ is the distance from robot to goal at time step $k$. In addition, $v_{x}(k)+\tau_{h}^{u b} a_{x}^{u b}$ is the fastest speed that the mobile robot can achieve with the maximum acceleration within the time horizon upper bound $\tau_{h}^{u b}$ which will be discussed below. Note that, due to $\omega_{\tau}$, the motion controller cannot generate a trajectory at time $k$ whose end point reaches a goal with the $\tau_{h \_t e m p}$.

Then, the motion controller compares $\tau_{h}$ with $\tau_{h-t e m p}$ to choose the larger one as $\tau_{h \_p r e d}$ as follows.

$$
\tau_{h \_p r e d}=\max \left(\tau_{h}, \tau_{h \_t e m p}\right) \text {. }
$$

The time horizon $\tau_{h \_p r e d}$ in (11) will be used for the trajectory prediction by the MPC in (1). If $\tau_{h_{-} t e m p}$ is greater than the $\tau_{h}$, i.e., $\tau_{h \_p r e d}=\tau_{h \_t e m p}$, we can predict trajectory long enough to find an optimal trajectory easily. Note that $\tau_{h_{-} t e m p}$ does not allow the end of predicted trajectory to reach a goal since $\omega_{\tau}$ in (10) is less than 1 . Therefore, at time $k$, if the optimal speed is the fastest speed with $\tau_{h}$, the robot still chooses the fasted speed as the optimal command even with $\tau_{h_{-}}$temp. It only 
enables easy finding of optimal commands without influencing the transient response intended. On the other hand, if $\tau_{h}$ is greater than $\tau_{h-t e m p}$, it means that the mobile robot is already so close to a goal that the small prediction time horizon $\tau_{h}$ can be used to search for an optimal trajectory.

In order to apply $\tau_{h \_p r e d}$ to trajectory prediction, an upper bound should be considered. If the mobile robot is far away from a goal, the $\tau_{h_{-} \text {temp }}$ can be extremely large, and it can cause a huge amount of computations for an extremely long trajectory prediction. Therefore, we should impose an upper bound $\tau_{h}^{u b}$ on $\tau_{h \_t e m p}$ with a reasonably large value, e.g., 3.5 second. Then, the final form of the adaptive prediction time horizon $\tau_{h \_ \text {pred }}$, which is used for trajectory prediction in the proposed motion controller, is expressed as follows.

$$
\tau_{h \_ \text {pred }}=\max \left(\tau_{h}, \min \left(\tau_{h \_t e m p}, \tau_{h}^{u b}\right)\right) .
$$

\section{Simulation Results}

In this section, the proposed motion controller will be evaluated in simulation using a Robot Operating System (ROS) environment. The dynamic object following software stack is implemented as an extension of the ROS navigation stack [14] with the motion controller algorithm being replaced by the one proposed in this paper. As a result of using this framework, the Path Planner [15] in Fig. 5 plans a path from a mobile robot to a goal with a fast interpolated navigation function on 2-dimensional costmaps created by the costmap_2d ROS package [16].

In the simulation, we use an ideal nonholonomic mobile robot platform on a 2-dimensional surface to verify performance of the proposed motion controller without extrinsic influences, e.g., roll motion and time lagging of actuator. Therefore, the 2-dimensional mobile robot platform is imple-

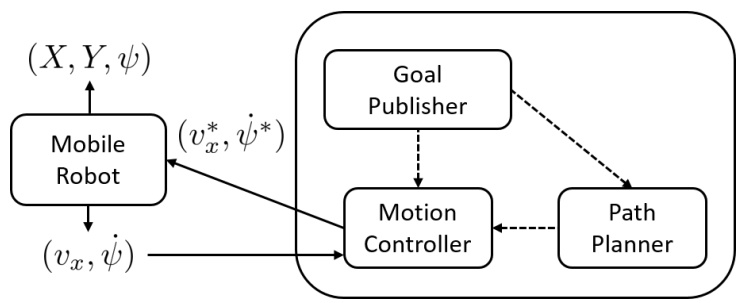

Figure 5: Motion Control for Dynamic Object Following

mented as a ROS node, as shown in Fig. 5, based on the dynamic vehicle model in (2) with $v_{y}=0$ and $\dot{v}_{y}=0$. The mobile robot in simulation takes in the optimal commands $v_{x}^{*}$ and $\dot{\psi}^{*}$ computed by the proposed motion controller and yields the pose $(X, Y, \psi)$ in the global frame along with its velocity states $v_{x}$ and $\dot{\psi}$. The states of mobile robot are updated every 0.05 seconds. The velocity states $v_{x}$ and $\dot{\psi}$ are provided to the motion controller as feedback signals. A dynamic object is simulated through the Goal Publisher in Fig. 5 that publishes goals moving with specified velocities every 0.1 seconds. Note that a localization algorithm does not run in the proposed following software stack since a prior map is not necessary for following a dynamic object in this paper. The parameters for the motion controller are listed in Table I, and the motion control algorithm computes the control commands at an update frequency $5 \mathrm{~Hz}$. Fig. 6 presents the magnitude of maximum lateral acceleration, $\left|\bar{a}_{y}\right|$, allowed for the longitudinal velocity $v_{x}$. The maximum lateral acceleration $\bar{a}_{y}$ is calculated as $\bar{a}_{y}=\operatorname{sign}\left(a_{y}\right)\left|\bar{a}_{y}\right|$. As shown in Fig. 6, the profile of $\left|\bar{a}_{y}\right|$ is defined as a piecewise linear function and its value decreases as $v_{x}$ increases to ensure cornering safety, e.g., rollover prevention.

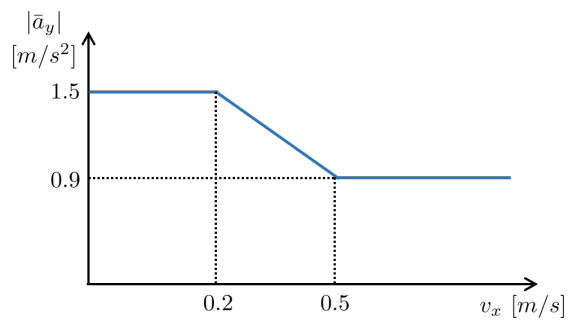

Figure 6: Magnitude of maximum lateral acceleration $\bar{a}_{y}$

Table I: Motion Controller Parameters

\begin{tabular}{cc|cc}
\hline \hline Parameter & \multicolumn{1}{c}{ Value } & Parameter & Value \\
\hline$p_{x}$ & 0.2 & $\omega_{o}$ & 0.0001 \\
$p_{\psi}$ & 0.2 & $\omega_{a y}$ & 1.0 \\
$\tau$ & $0.1[\mathrm{~s}]$ & $\omega_{g}$ & 0.001 \\
$\ddot{\psi}^{u b}$ & $4.5[\mathrm{rad} / \mathrm{s}]$ & $\omega_{p}$ & 0.01 \\
$\ddot{\psi} l b$ & $-4.5[\mathrm{rad} / \mathrm{s}]$ & $n_{\psi}$ & 60 \\
$a_{x}^{u b}$ & $0.5\left[\mathrm{~m} / \mathrm{s}^{2}\right]$ & $n_{v x}$ & 30 \\
$a_{x}^{l b}$ & $-0.5\left[\mathrm{~m} / \mathrm{s}^{2}\right]$ & & \\
\hline \hline
\end{tabular}

\section{A. Prediction Time Horizon as Time Constant}

As discussed previously, with the proposed motion control based on the downscaled MPC, the prediction time horizon $\tau_{h}$ acts as the time constant, and therefore we can tune $\tau_{h}$ to adjust the rate of convergence to a goal. This finding can be verified though a simulation with different $\tau_{h}$ values. In the simulation, a goal is static and located at $3 \mathrm{~m}$ in front of the mobile robot for the four different $\tau_{h}$ values, 1.5, 2.0, 2.5, and 3.0 seconds.

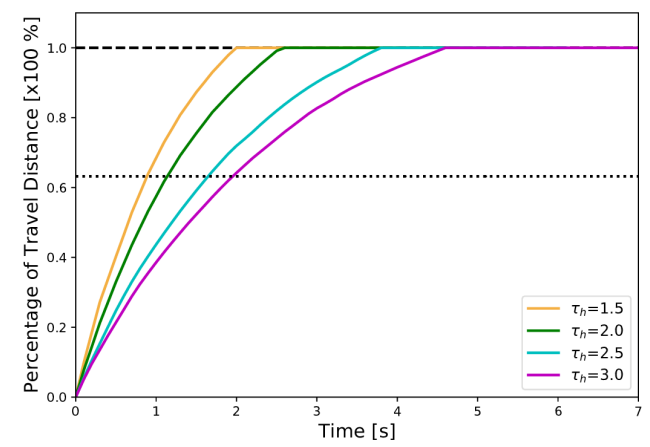

Figure 7: Different rate of convergence to a goal due to different prediction time horizon

As shown in Fig. 7, with the smaller $\tau_{h}$, the mobile robot accelerates $v_{x}$ harder to reach the goal faster. The time steps 
when the mobile robot navigates $63.2 \%$ of the distance to goal are around $0.9,1.2,1.7$, and 2.0 seconds, respectively. The values of $\tau_{h}$ used for simulation do not match the measured time steps exactly, but this simulation demonstrates that $\tau_{h}$ can be used to adjust the rate of convergence to a goal.

In the simulation presented in Fig. 8, the mobile robot follows a moving object with two different prediction time horizons $\tau_{h}$. The motion of the object is intentionally limited to 1 dimensional motion, i.e., straight line, to highlight the effect of the prediction time horizon. The positions of the moving object are published as goals in the ROS environment. The speed of the object is $0.0 \mathrm{~m} / \mathrm{s}$ at first, and it reaches $1.0 \mathrm{~m} / \mathrm{s}$ immediately after around 2.0 seconds has passed. This abrupt speed change simulates following a highly dynamic object, e.g., a human.

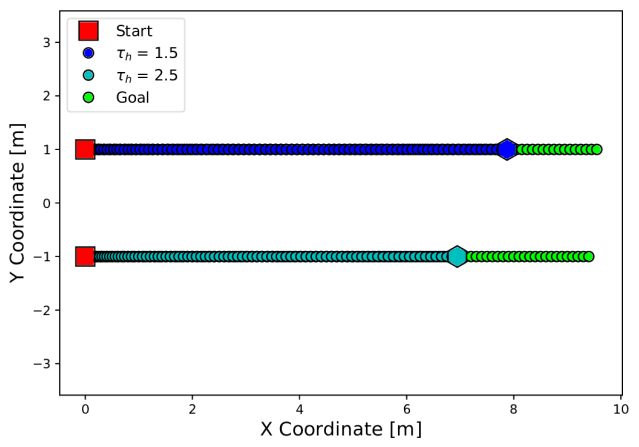

Figure 8: Following a highly dynamic object with different prediction time horizon

In Fig. 8, the blue and cyan circles represent the positions of the mobile robot for $\tau_{h}=1.5$ and $\tau_{h}=2.5$, respectively. The mobile robot with $\tau_{h}=2.5$ falls behind the one with $\tau_{h}=1.5$ at the end of simulation. This is because the velocity $v_{x}$ of the mobile robot increases faster to catch up with the dynamic goal more quickly with $\tau_{h}=1.5$ than $\tau_{h}=2.5$, as shown in Fig. 9.
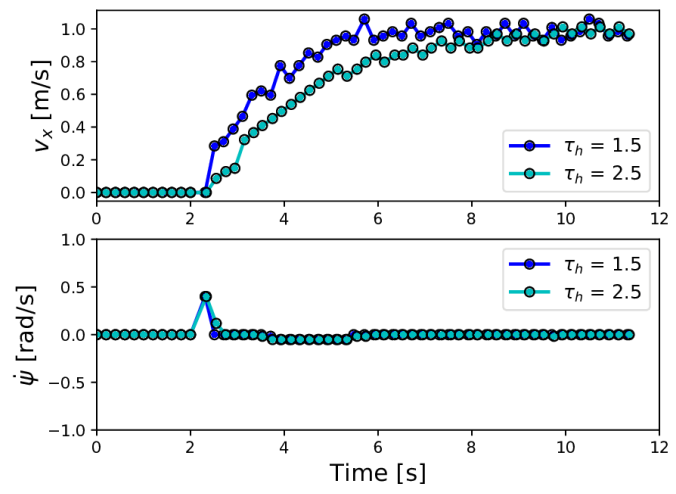

Figure 9: Longitudinal velocity $\left(v_{x}\right)$ and yaw rate $(\dot{\psi})$ generated with different prediction time horizon

\section{B. Adaptive Prediction Time Horizon}

The previous simulation has verified that the prediction time horizon $\tau_{h}$ can decrease to react quickly to movement of a dynamic object. But, the small $\tau_{h}$ can make motion controller vulnerable to uncertainties in choosing optimal motion control commands. Therefore, this paper proposes the adaptive prediction time horizon which varies depending on the distance to a goal.

The following simulation results present how the proposed approach contributes to the robustness improvement. In Fig. 10 , the prediction time horizon $\tau_{h}$ is set to a small value of 0.5 second, and the initial orientation of the mobile robot is to the right at the start location in Fig. 10. Without the proposed

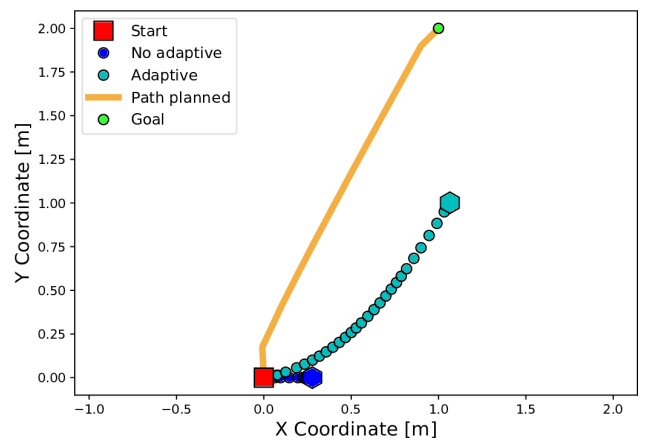

Figure 10: Reaching a goal with adaptive prediction time horizon

adaptive prediction time horizon, in Fig. 10, the mobile robot (blue circle) chooses a narrow-sighted trajectory to just go straight and deviates from the planned path. Furthermore, the mobile robot is stuck as it continues to try turning left and right repeatedly, as $\dot{\psi}$ presents in Fig. 11. However, as shown in Fig. 10, the mobile robot with $\tau_{h}$ replaced by $\tau_{h \_ \text {pred }}$ in (12) heads for the goal without difficulty of searching for optimal control commands.

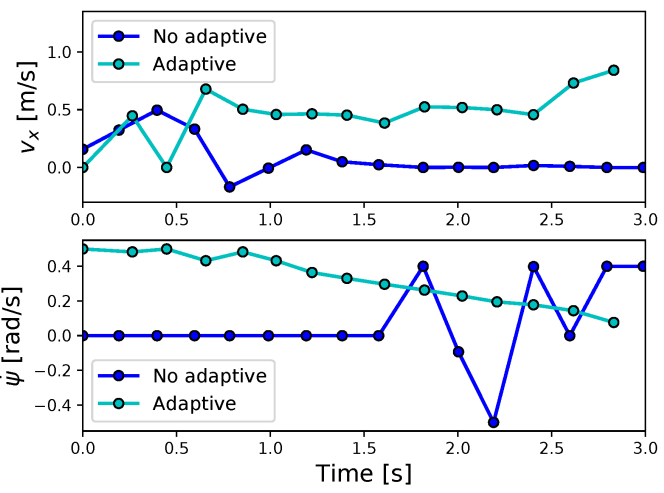

Figure 11: Longitudinal velocity $\left(v_{x}\right)$ and yaw rate $(\dot{\psi})$ generated with adaptive prediction time horizon 


\section{Tight Path Following}

The proposed motion controller takes tight path following into consideration through the cost term in $(3 \mathrm{~d})$. The planned path serves as guidance for motion control algorithm, and therefore it improves the robustness of motion controller. In particular, if the mobile robot needs to avoid obstacles, following a path planned to a goal can exhibit the benefit clearly by, e.g., preventing mobile robots stuck in oscillating motion. The tightness of the path following can be adjusted by tuning the weight $\omega_{p}$. Fig. 12 presents the trajectories of the mobile robot and goal of a simulation in which the mobile robot moves straight first and starts taking left turn to follow the dynamic goal with $v_{x}=2.0 \mathrm{~m} / \mathrm{s}$ and $\dot{\psi}=0.7 \mathrm{rad} / \mathrm{s}$. In order to verify the performance of tight path following, the safe maneuver cost term in (3b) is set to zero. In Fig. 12, the orange

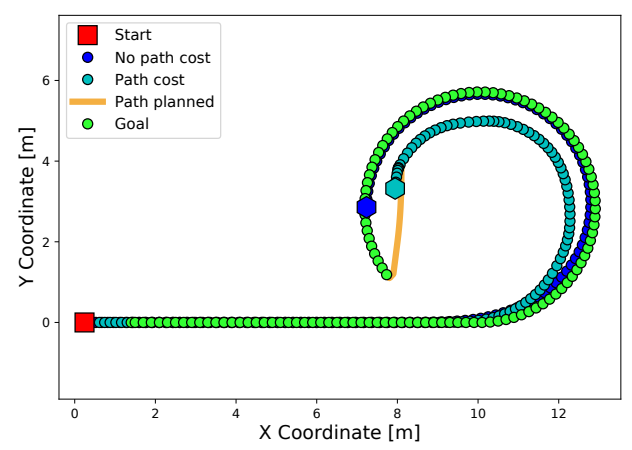

Figure 12: Following a dynamic object with tight path following considered

line represents the last path in the simulation with $\tau_{h}=2.5$ that is planned from the mobile robot to the moving goal. The blue circles represent the positions of the mobile robot without the path following term in $(3 \mathrm{~d})$, and therefore, the mobile robot mostly moves over the points that the dynamic goal has passed. But, with the tight path following cost term, the mobile robot, cyan circles in Fig. 12, takes a smaller turn than without considering tight path following since it tries to follow the planned path which is almost a straight line to the goal.

\section{Safe Cornering}

The lateral acceleration $a_{y}$ is included as a cost term in (3b) to generate safe maneuvers for the mobile robot while taking turns. In the simulation with $\tau_{h}=2.5$ in Fig. 13, the mobile robot undergoes the same scenario as in Fig. 12. The tight path following cost term is used, and therefore the mobile robot tries to create a sharper turn than the dynamic object.

In Fig. 13, the trajectory of the mobile robot with the $a_{y}$ cost term in (3b) is similar to the one without it. But, with the safe cornering maneuver considered, the mobile robot slows down to decrease the velocity $v_{x}$ after about 5 seconds although it generates a similar yaw rate $\dot{\psi}$, as shown in Fig. 14 .

\section{EXPERIMENTAL RESULTS}

In the simulation, the proposed motion controller is implemented in a ROS framework and the mobile robot is modeled

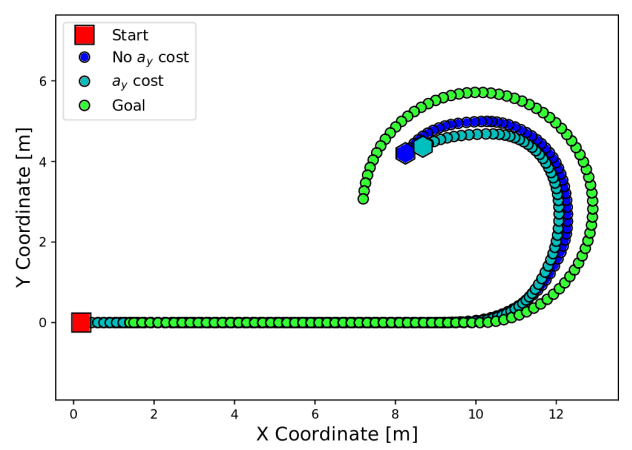

Figure 13: Following a dynamic object with mobile robot's safe cornering considered

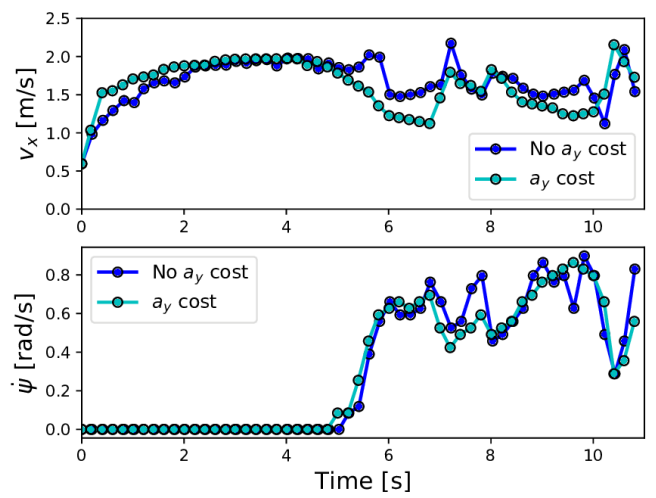

Figure 14: Longitudinal velocity and yaw rate generated when considering mobile robot's safe cornering

as a separate ROS node. Therefore, experimental tests can be conducted by simply replacing the Mobile Robot in Fig. 5 with a real mobile robot platform.

\section{A. Mobile Robot Platform}

The test robot is built on the Clearpath Jackal differentialdrive wheeled platform shown in Fig. 15. The base platform receives linear and angular velocity commands and provides odometry data from wheel encoders. The platform is outfitted with additional Lidar sensors. An RPLidar A3 2D Lidar is mounted on the front bumper and is used for obstacle detection. A Velodyne VLP-32C 3D Lidar is mounted on top and is used for target tracking.

\section{B. Human Tracking and Goal Broadcasting}

In the experimental tests, a human tracking algorithm replaces the Goal Publisher in Fig. 5 to broadcast goals for the proposed motion controller. Human tracking is performed using the Leg Tracker ROS Package that is based on the work in [7]. A 2D laserscan is generated from the Velodyne and passed to the leg tracker ROS node. The leg tracker maintains unique labels for each human around the robot. The robot can be assigned a specific label ID to follow as its target. A direction vector is computed between the $2 \mathrm{D}$ pose of the 


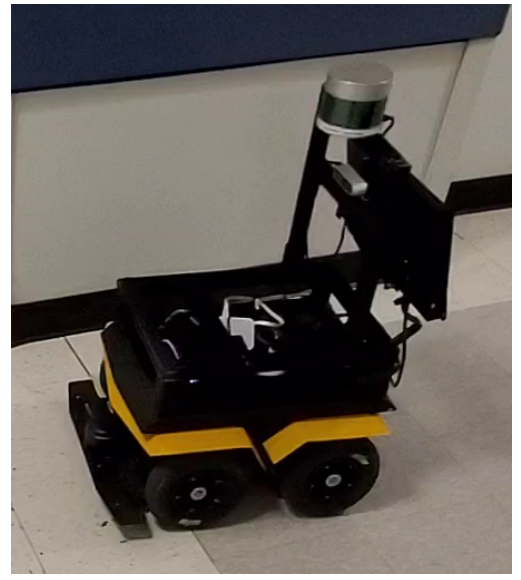

Figure 15: Test robot consisting of a Clearpath Jackal base with an RPLidar A3 on the front bumper and a Velodyne VLP32C 3D Lidar on top.

target and the 2D pose of the robot within the global frame, i.e., the odometry frame in the ROS navigation stack. A goal is assigned as the location along that vector that is offset $1 \mathrm{~m}$ behind the target. Goals are repeatedly generated and provided to the motion controller at a rate of $7.5 \mathrm{~Hz}$

\section{Following Performance}

Tests with the real mobile robot platform also utilize the same control update frequency $5 \mathrm{~Hz}$ and parameters listed in Table I as in the Simulation Results except for $\tau=0.2$ second. The tests demonstrate the finding that the prediction time horizon acts as the time constant. In Fig. 16, the percentage of travel distance is presented when the mobile robot is commanded to navigate to a static goal in front of it. The time steps when the mobile robot navigates $63.2 \%$ of the distance are around 1.7, 2.5, and 3.2 seconds for $\tau_{h}=2.5, \tau_{h}=3.5$, and $\tau_{h}=4.5$, respectively. This implies that the prediction time horizon has a linear relationship with the time constant.

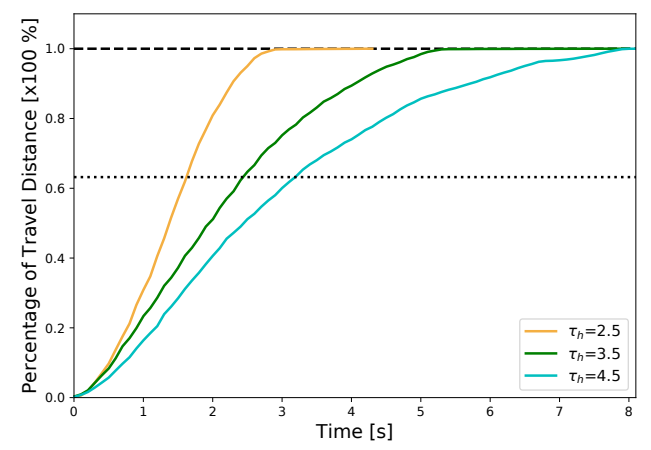

Figure 16: Different rate of convergence to a goal due to different prediction time horizon

To validate the collision avoidance and following performance, the mobile robot was assigned to follow a person who is going over an obstacle, performing a zigzag maneuver, and turning around. In this test, the motion controller computed the following control commands with the prediction time horizon $\tau_{h}=2.5$. As shown in the red box in Fig. 17a, the person walked in a straight-line path stepping over an obstacle while the mobile robot deviates from the person's trajectory to go around that obstacle. The gray dots in Fig. 17a and Fig. 17b represent occupied grid points in the costmap used in motion control. After avoiding collision with the obstacle, the mobile robot follows the person walking in a zigzag motion. In Fig.

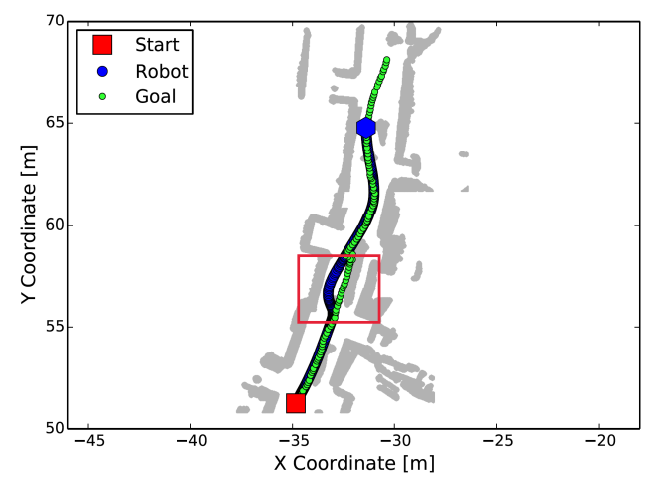

(a) Avoiding collision with an obstacle and following a person performing a zigzag maneuver

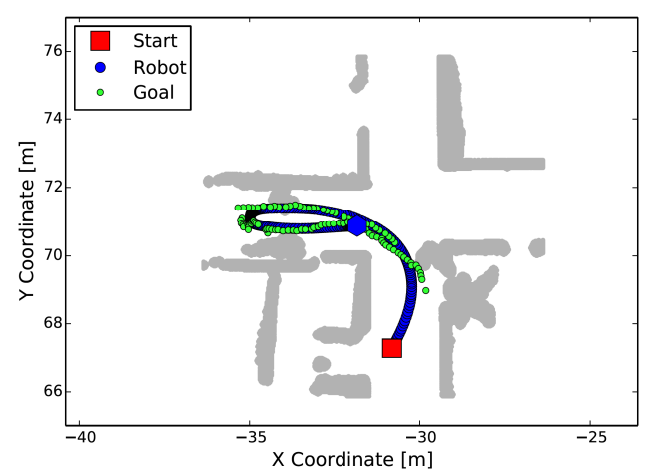

(b) Following a person turning around in a tight space

Figure 17: Obstacle Avoidance and Following A Person

$17 \mathrm{~b}$, the mobile robot follows the person turning around in a tight space.

In addition, since the mobile robot takes safe maneuvers into consideration, it slows down if a sharp turn is required to follow the person, as shown in Fig. 18. The longitudinal velocity $v_{x}$ decreases when the magnitude of yaw rate $\dot{\psi}$ increases significantly in the red boxes.

\section{CONCLUSION}

This paper proposes a motion controller to follow a dynamic object based on downscaled MPC. It is proved theoretically that the prediction time horizon acts as a time constant to determine the transient response in the proposed MPC formulation. Therefore, the proposed motion control algorithm can adapt to the dynamics of object by tuning the prediction time horizon. To ensure robustness when following an object with a small prediction time horizon, this paper also proposed to introduce 


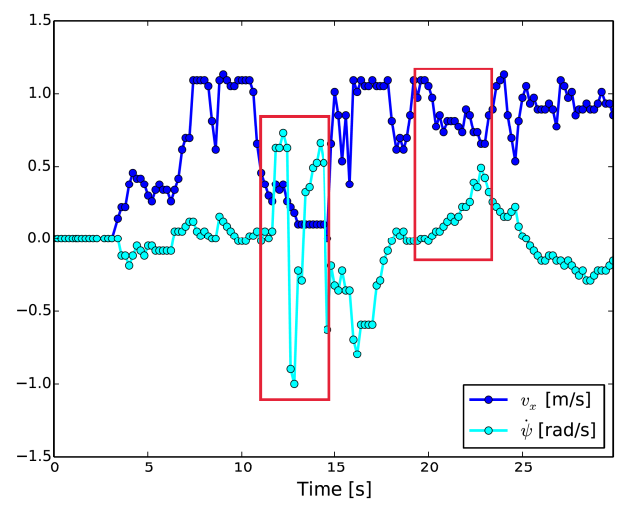

Figure 18: Longitudinal velocity and yaw rate generated when a sharp turn is required to follow a person

the adaptive prediction time horizon. The cost function used in the motion controller takes rollover prevention, collision avoidance, and tight path following into consideration. The proposed motion controller were demonstrated through extensive simulation and experiments of a test mobile robot.

\section{REFERENCES}

[1] B. Lee, J. Choi, C. Baek, and B. Zhang, "Robust human following by deep bayesian trajectory prediction for home service robots," in 2018 IEEE International Conference on Robotics and Automation (ICRA), May 2018, pp. 7189-7195.

[2] G. Doisy, A. Jevtic, E. Lucet, and Y. Edan, "Adaptive person-following algorithm based on depth images and mapping," in In Workshop on Robot Motion Planning: Online, Reactive, and in Real-time, Proceedings of the IEEE International Conference on Intelligent Robots and Systems (IROS), 2012.

[3] P. Morin and C. Samson, "Motion control of wheeled mobile robots," in Springer Handbook of Robotics, January 2008, pp. 799-826.

[4] H. S. Shanee, K. Dror, O. Tal, and E. Yael, "The influence of following angle on performance metrics of a human-following robot," in 2016 25th IEEE International Symposium on Robot and Human Interactive Communication (RO-MAN), August 2016, pp. 593-598.

[5] Zhichao Chen and S. T. Birchfield, "Person following with a mobile robot using binocular feature-based tracking," in 2007 IEEE/RSJ International Conference on Intelligent Robots and Systems, Oct 2007, pp. $815-820$.

[6] N. A. Olmedo, H. Zhang, and M. Lipsett, "Mobile robot system architecture for people tracking and following applications," in 2014 IEEE International Conference on Robotics and Biomimetics (ROBIO 2014), Dec 2014, pp. 825-830.

[7] A. Leigh, J. Pineau, N. Olmedo, and H. Zhang, "Person tracking and following with 2d laser scanners," in 2015 IEEE International Conference on Robotics and Automation (ICRA), May 2015, pp. 726733.

[8] D. Fox, W. Burgard, and S. Thrun, "The dynamic window approach to collision avoidance,' IEEE Robotics Automation Magazine, vol. 4, no. 1, pp. 23-33, March 1997.

[9] B. P. Gerkey and K. Konolige, "Planning and control in unstructured terrain," in In Workshop on Path Planning on Costmaps, Proceedings of the IEEE International Conference on Robotics and Automation (ICRA), 2008.

[10] R. Rajamani, Vehicle Dynamics And Control. Springer Verlag, 2006.

[11] P. E. Hart, N. J. Nilsson, and B. Raphael, "A formal basis for the heuristic determination of minimum cost paths," IEEE Transactions on Systems Science and Cybernetics, vol. 4, no. 2, pp. 100-107, July 1968.

[12] P. J. Antsaklis and A. N. Michel, Linear systems. McGraw-Hill, 1997.

[13] K. Ogata, Modern Control Engineering, ser. Instrumentation and controls series. Prentice Hall, 2010. [Online]. Available: https://books.google.com/books?id=Wu5GpNAelzkC
[14] ROS.org. Navigation stack. [Online]. Available: http://wiki.ros.org/navigation

[15] - Navigation stack - navfn. [Online]. Available: http://wiki.ros.org/navfn

[16] - Navigation stack - costmap 2d. [Online]. Available: http://wiki.ros.org/costmap $2 \mathrm{~d}$ 